\title{
Enhancement of cooling design on the performance of thermoelectric Generator
}

\author{
Changqing Guo ${ }^{1, a^{*}}$, Changfeng Yan ${ }^{1, b}$ and Xiaoyong Zhao ${ }^{2, c}$ \\ ${ }^{1}$ Guangzhou Institute of Energy Conversion, Chinese Academy of Sciences, Guangzhou, China \\ ${ }^{2}$ University of Chinese Academy of Sciences, Beijing, China \\ aguocq@ms.giec.ac.cn, byancf@ms.giec.ac., czhaoxy@ms.giec.ac.cn
}

Keywords: Thermoelectric generator; Jet impinging cooling; Numerical simulation.

\begin{abstract}
Thermoelectric generators(TEG) is considered as a promising technology for utilizing low-grade thermal energy, while the cooling system plays a key role in TEG. A two-dimensional numerical model of jet impinging jet cooling is developed based on the commercial code FLUENT. The influences of several factors on thermoelectric conversion efficiency are observed. The factors include the air inlet velocity, the TE legs height, and the fins. The results show that the air inlet velocity is one of the most important factors for the cooling performance, the convection heat transfer efficiency and conversion efficiency significantly increases with the improvement of the air velocity. Higher TE legs height can influence the TEG performance effectively, 5 7mm legs height is recommended in this study. And fins can effectively increase the TEG efficiency, the thermoelectric conversion efficiency with $1 \mathrm{~mm} \times 1 \mathrm{~mm}$ fins is $6.73 \%$ which was 2.4 times higher than without fins.
\end{abstract}

\section{Introduction}

With the increase of the global energy consumption, energy saving and emissions have become world wide problems to global sustainable development. Hendricks and Choate ${ }^{[1]}$ reported that about $1 / 3$ of the manufacturing industrial energy was discharged directly to the atmosphere or cooling systems as heat. With development of waste heat recovery technologies, the high temperature waste heat have already been utilized to generate electricity or heat to increase the energy utilization efficiency, while most of this lost energy is "low quality" and not practical or economical to recover, in order to expand the waste heat recovery to low grade waste heat, a new type of thermoelectric generators (TEG) based on Seebeck effect has become a popular research topic recently which can convert thermal energy into electricity directly without any moving components. TEG technology have been proposed for some applications such as solar energy, Geothermal and some sort of industrial waste heat.

As a green technology, TEG have showed advantages in low grade waste heat recovery ${ }^{[2-4]}$, Compared with the conventional methods, TEG technology has the advantages of compact, quiet, highly reliable and environmentally friendly, and the advancements in thermoelectric materials with high figure of merit have highlighted the TEG's energy efficiency and commercial potential.

The most widely study of TEG technology is used for automotive exhaust gas, Bass et al. ${ }^{[5-6]}$ applied an array with 72 pieces of TEG on a diesel truck. and energy conversion efficiency of $4.5 \%$ was achieved when the temperature of hot and cold sides of TEGs maintaining $230{ }^{\circ} \mathrm{C}$ and $30{ }^{\circ} \mathrm{C}$ respectively, Thacher et al. ${ }^{[7]}$ attached HZ-20 $\left(\mathrm{Bi}_{2} \mathrm{Te}_{3}\right)$ to a light truck engine. The whole system can produce up to $330 \mathrm{~W}$ power. In addition, Champier and co-workers ${ }^{[8]}$ applied TE modules on biomass cook stoves to generate electricity to power the fan and light. Crane et al. ${ }^{[9]}$ used hot water as a heat source and air as a heat sink to simulate an exhaust thermoelectric generator.

Dan et al. ${ }^{[10]}$ presented a liquid metal based TEG system which had the efficiency of $2 \%$. Gou et al. ${ }^{[11]}$ proposed some advices, like increasing the waste heat temperature, expanding heat sink surface area in proper range and enhancing cold-side heat transfer capacity, to enhance the TEG performance. 
As the source that TEG utilizes is low grade waste heat, cheap or free, and there is no consume of fresh fuel for electricity production, it will be able to obtain additional benefits in terms of an improved overall efficiency. In addition, the energy conversion efficiency is quite attractive when the TEG works in a parasitic mode.

According to the Seebeck effect of thermoelectric, the higher the temperature difference between hot and cold side of the TE material, the power generation is higher which means the heat transfer plays an important role in the TEG system. The hot side is usually determined by the waste heat resources, so in order to increase the temperature difference, high heat transfer performance should get to reduce the temperature at the cold side as lower as possible. Effective exhaust heat exchanger design is critical for improving TEG performance, and some novel types of heat exchanger structures, such as roll cake structure $^{[12]}$, multi-layer plates ${ }^{[13]}$, microchannels ${ }^{[14]}$, and heat pipes ${ }^{[15]}$ have been used for TEG. In this study , a Jet Impinging Cooling of TEG is studied.

\section{Mathematical model and control equations}

\section{Physical model}

A TEG module is composed by some pairs of N-legs and P-legs that are thermally connected in parallel and electrically connected in series by metal electrodes. The simplified 2D physical model of jet impinging cooling TEG is shown in Fig. 1 . In this study $\mathrm{Bi}_{2} \mathrm{Te}_{3}$-based thermoelectric materials was used.

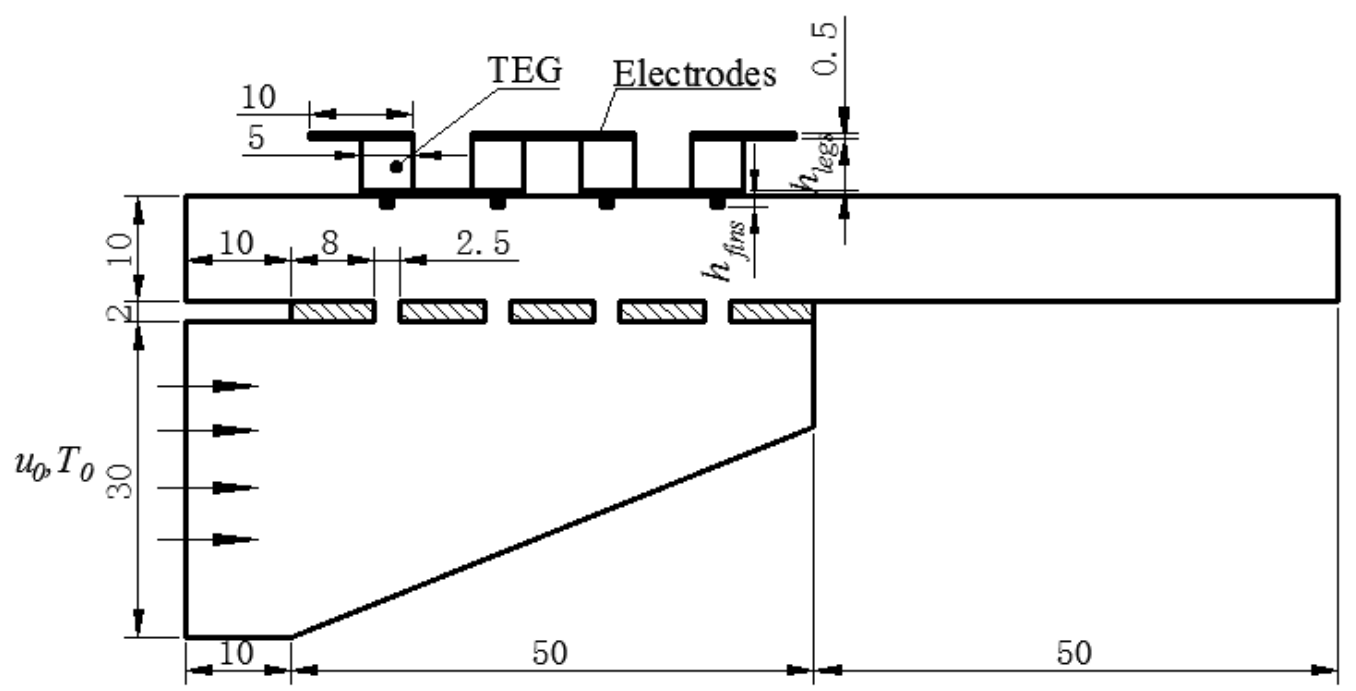

Fig.1 Physical model of TEG simulation (unit in $\mathrm{mm}$ )

The relevant parameters used are provided in Table 1.

Table 1 Operating conditions and model parameters used in simulations

\begin{tabular}{cccc}
\hline Parameters & Unit & symbol & Values \\
\hline Air inlet velocity & $\mathrm{m} / \mathrm{s}$ & $u_{0}$ & $3, \mathbf{5}, 10,15$ \\
Air inlet temperature & $\mathrm{K}$ & $T_{0}$ & 300 \\
TEG legs height & $\mathrm{mm}$ & $h_{\text {legs }}$ & $3, \mathbf{5}, 7,9$ \\
Cold side fin height & $\mathrm{mm}$ & $h_{\text {fins }}$ & $\mathbf{0 , 1}$ \\
TEG Hot side temperature & $\mathrm{K}$ & $T_{h}$ & 600 \\
\hline
\end{tabular}

The boldface values represent nominal parameters.

\section{Mathematical model}

The TEG cooling process can be described by the following equations of continuity, momentum, and energy closed by the realizable $k-\varepsilon$ turbulence model. 
Continuity equation: $\frac{\partial}{\partial x_{i}}\left(\rho u_{i}\right)=0$

Momentum equation: $\frac{\partial}{\partial x_{j}}\left(\rho u_{i} u_{j}\right)=-\frac{\partial p}{\partial x_{i}}+\frac{\partial}{\partial x_{j}}\left(\left[\mu\left(\frac{\partial u_{i}}{\partial x_{j}}+\frac{\partial u_{j}}{\partial x_{i}}\right)\right]-\frac{2}{3} \mu \frac{\partial u_{l}}{\partial x_{l}} \delta_{i j}\right)$

Energy equation: $\nabla \cdot\left(\rho c_{p} T\right)=\nabla \cdot\left(\lambda_{e} \nabla T\right)$

(3)

In the solid region like TEG legs, electrodes and middle plates, the energy equation can be written as:

$$
\frac{\partial}{\partial x_{i}}\left(k \frac{\partial T}{\partial x_{i}}\right)+S_{E}=0
$$

Where $k$ is the thermal conductivity of TEG legs, electrodes or middle plates, For electrodes and middle plates, the energy source term $S_{E}=0$, while for TEG legs, the source term of Joule heat can be written as

$$
S_{E}=\frac{I^{2} \rho}{A^{2}}
$$

And the electromotive force $E$ and electric current $I$ is

$$
\begin{aligned}
& E=\int_{T_{c}}^{T_{h}}\left(\alpha_{P}(T)-\alpha_{N}(T)\right) d T \\
& I=\frac{E}{R_{L}+R_{P-l e g}+R_{N-l e g}}
\end{aligned}
$$

Where $\alpha$ is Seebeck coefficient and $\rho$ is the electrical resistivity. $T_{h}$ and $T_{c}$ is the temperature at the hot and cold side respectively, and $R_{L}, R_{P-l e g}$ and $R_{N-l e g}$ is the load resistance, P-legs resistance and N-legs resistance respectively, the leg resistance of TE legs can be calculated as follows:

$$
R_{\text {leg }}=\sum \int_{0}^{h_{T E}} \frac{\rho}{A} d y
$$

It has been found that the model based on variable TE properties is superior to that based on constant material properties ${ }^{[16]}$. The variable TEG Seebeck coefficient, thermal conductivity and electrical resistivity are listed in Table 2.

Table 2 The variable TE material properties

\begin{tabular}{ccc}
\hline Parameters & Unit & Equations \\
Seebeck coefficient & $\mu \mathrm{V} / \mathrm{K}$ & $\alpha_{p}=-490.14+4.42 T-8.24 \times 10^{-3} T^{2}+4.03 \times 10^{-6} T^{3}$ \\
& & $\alpha_{n}=351.37-3.74 T+7.62 \times 10^{-3} T^{2}-4.51 \times 10^{-6} T^{3}$ \\
Thermal conductivity & $\mathrm{W} /(\mathrm{m} \cdot \mathrm{K})$ & $\begin{array}{l}k_{p}=9.88-5.79 \times 10^{-2} T+1.21 \times 10^{-4} T^{2}-7.27 \times 10^{-8} T^{3} \\
k_{n}=11.42-6.86 \times 10^{-2} T+1.49 \times 10^{-4} T^{2}-9.53 \times 10^{-8} T^{3}\end{array}$ \\
& & $\begin{array}{l}\rho_{p}=2.75 \times 10^{-5}-2.42 \times 10^{-7} T+8.47 \times 10^{-10} T^{2}-7.74 \times 10^{-13} T^{3} \\
\text { Electrical resistivity }\end{array}$ \\
& $\Omega \cdot \mathrm{m}$ & $\rho_{n}=5.08 \times 10^{-7}-4.84 \times 10^{-9} T+1.93 \times 10^{-10} T^{2}-2.53 \times 10^{-13} T^{3}$ \\
\hline
\end{tabular}

The subscript $p$ and $n$ means the TEG P-legs and N-legs respectively.

\section{Boundary conditions}

Boundary conditions are expressed as follows:

(1) Inlet: $T=T_{i n}, u=u_{0}$

(2) Outlet: $P=P_{0}, \frac{\partial \dot{v}}{\partial x}=\frac{\partial T}{\partial x}=0$

(3) Hot side of TEG legs: $T=600 \mathrm{~K}$ 


\section{Numerical method}

The commercial software FLUENT (Version 6.3) based on finite-volume method is utilized to solve all of the governing equations, together with the boundary conditions. The SIMPLE algorithm is used for the pressure-velocity coupling. The inlet boundary condition is velocity inlet. And the outlet is modeled as a pressure outlet. The $\mathrm{C}$ codes of the source terms were compiled and linked with FLUENT by user defined function (UDF).

The model was meshed using the quadrilateral grids. The mesh near the wall were refined using boundary layer and $y^{+}$of the first grid point is less than unity. And mesh independence tests have been performed to ensure that the solution does not vary with the number of computational elements,

The important characteristics for TEG modules are the thermoelectric conversion efficiency which is defined as follows:

$\eta=\frac{P_{0}}{Q_{h}} \times 100 \%=\frac{I^{2} R_{L}}{\left(\alpha_{P}-\alpha_{N}\right) I T_{h}-0.5 I^{2} R_{i}+\int_{T_{c}}^{T_{h}}\left(\frac{A_{P} k_{P}}{h_{T E}}+\frac{A_{N} k_{N}}{h_{T E}}\right) d T} \times 100 \%$

\section{Results and discussion}

\section{Velocity vector and temperature field}

Fig. 2 shows the temperature field and the velocity vector. The velocity vector and temperature field of TEG cooling process when the inlet velocity is $10 \mathrm{~m} / \mathrm{s}$ and TEG hot side temperature is $600 \mathrm{~K}$. It can be seen that air velocity increased with the decrease of the section area along the $x$ direction, and the jets create when the air flow through the holes which made the air velocity near the TEG cold side larger than the inlet velocity and make a more effective heat transfer performance. And the flow boundary layer and thermal boundary layer can be found near the TEG cold side which means that improving the flow and heat tranfer performance near the cold side can get more higher TEG conversion efficiency.

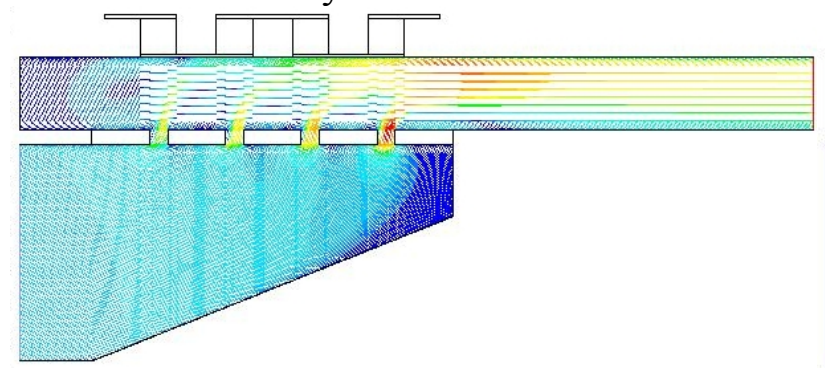

(a) Velocity vectors

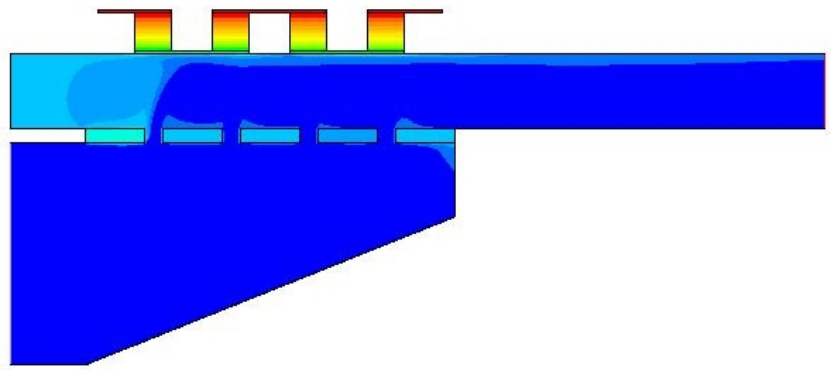

(b) Temperature field

Fig. 2 The velocity vectors and temperature field

\section{Effect of inlet velocity}

For jet impinging cooling, the inlet velocity play a key role in the TEG conversion efficiency because it can influence the TEG cold side temperature. Fig. 3 shows the result with different inlet velocity ranged from $3 \mathrm{~m} / \mathrm{s} \sim 15 \mathrm{~m} / \mathrm{s}$ while the TEG leg height $\left(h_{\text {legs }}\right)$ is $5 \mathrm{~mm}$. From Fig. 3 , the cold side temperature decreased and the thermoelectric conversion efficiency increased with the increase of inlet velocity. Higher velocity generates higher turbulence and thiner flow and thermal boundary layer, and then results in higher convection heat transfer coefficient and higher conversion efficiency. However, the improvement of applied inlet volcity is limited by driven power to overcome the pressure drop.

\section{Effect of TEG legs height}

The effect of TEG legs height is shown in Fig. 4. The plot shows that higher legs resulted in larger efficient conversion rate. This is because the thermoelectric materials can not be considered as heat insulator for the thermal conductivity of P-type and N-type materials is about $1.3 \sim 2.8 \mathrm{~W} /(\mathrm{m} . \mathrm{K})$ and $1.6 \sim 3.1 \mathrm{~W} /(\mathrm{m} . \mathrm{K})$ respectively at the temperature range of $30 \sim 300^{\circ} \mathrm{C}$. At the same cooling conditions, 
the shorter legs will lead to smaller temperature gradient in legs and lower conversion efficiency, while higher TEG legs needs more semiconductor material which means more cost and lower economics, so the suitable legs height of $5 \sim 7 \mathrm{~mm}$ is recommended in this study.

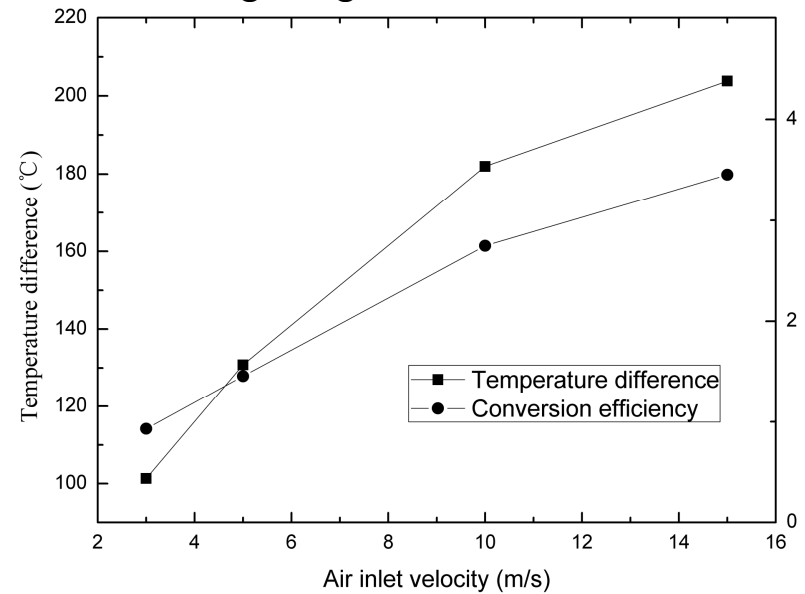

Fig.3 Effects of air inlet velocity

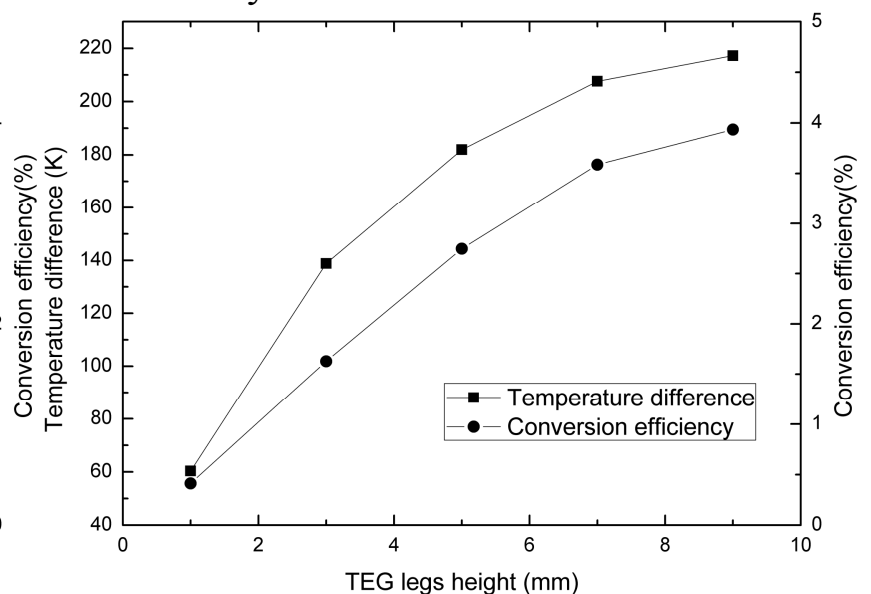

Fig. 4 Effects of TEG legs height

\section{Effect of Fins}

Finned surface are widely used in compact heat exchangers to improve heat transfer performance, usally it is considered that the role of fins is to increase heat transfer area, and effectively destroyed the boundary layer to enhance the ability of fluid to be turbulence. $1 \mathrm{~mm}$ height and $1 \mathrm{~mm}$ width fins are used to increase the cooling performance. The comparision between system with fins and without fins are listed in Table 3.

Table 3 The comparision of TEG performances between system with fins and without fins

\begin{tabular}{cccc}
\hline Systems & Cold side temperature & Temperature difference & Conversion efficiency \\
\hline Without fins & $145.1^{\circ} \mathrm{C}$ & $182.0^{\circ} \mathrm{C}$ & $2.75 \%$ \\
With fins & $43.9^{\circ} \mathrm{C}$ & $283.1^{\circ} \mathrm{C}$ & $6.73 \%$ \\
\hline
\end{tabular}

As seen in Table 2, using the fins on the electrodes can effecitively increase the performance of TEG. The cold side temperature at TEG legs droped from the $145.1^{\circ} \mathrm{C}$ to $43.9^{\circ} \mathrm{C}$ which means the temperature difference between the TEG hot sides and cold sides increased from $182.0^{\circ} \mathrm{C}$ to $283.1^{\circ} \mathrm{C}$, and the thermoelectric conversion efficiency with fins is $6.73 \%$ which was 2.4 times higher than that without fins.

\section{Conclusions}

This study developed a two-dimensional numerical model of TEG coupled with jet impinging cooling system. The effects of air inlet velocity, TEG legs height and fins surface were investigated. The conclusions can be drawn as below.

(1) The air inlet velocity plays a key role for cooling performance and TEG efficiency. The temperature difference between TEG hot and cold side and the conversion efficiency increased with the increase of air velocity.

(2) With the increase of TEG legs height, the temperature difference and efficiency is increased, but taking into account the cost and the economy, $5 \sim 7 \mathrm{~mm}$ height is recommended in this study.

(3) Finned surface can effectively enhance the heat transfer rate and then results in higher efficiency, and the thermoelectric conversion efficiency with $1 \mathrm{~mm} \times 1 \mathrm{~mm}$ fins was 2.4 times higher than without fins. 


\section{Acknowledgements}

The authors gratefully acknowledge the financial support of Guangzhou Science and Technology Project (No. 2013J4500027);Guangdong Science and Technology Project (No. 2013B050800007, No. 2014A020216030)

\section{References}

[1] Hendricks, T., Choate, W. T., Engineering Scoping Study of Thermoelectric Generator Systems for Industrial Waste Heat Recovery, Department of Energy, 2006.

[2] HoSung Lee. Optimal design of thermoelectric devices with dimensional analysis. Applied Energy.106(2013)79-88.

[3] C.T. Hsu, G.Y. Huang, H.S. Chu, B. Yu, D.J. Yao.Experiment and simulation on low-temperature waste heat harvesting system by thermoelectric power generators Applied Energy, 88 (2011). 1291-1297.

[4] L.E. Bell. Cooling, heating, generating power, and recovering waste heat with thermoelectric systems.Science, 321 (2008)1457-1461.

[5] Bass JC. Thermoelectric generator for motor vehicle. U.S. Patent US5625245.(1997)

[6] Bass JC, Elsner NB, Leavitt FA. Method for fabricating a thermoelectric module with gapless eggcrate. U.S. Patent US 5856210.(1999)

[7] E. Thacher, B. Helenbrook, M. Karri, C. Richter. Testing of an automobile exhaust thermoelectric generator in a light truck. J. Automob Eng, 221 (2007) 95-107.

[8] Champier D, Bedecarrats JP, Rivaletto M, Strub F. Thermoelectric power generation from biomass cook stoves. Energy, 35(2010)935-942.

[9] D.T. Crane, G.S. Jackson. Energy Convers Manag, 45 (2004)1565-1582.

[10] Dan Dai, Yixin Zhou, Jing Liu. Liquid metal based thermoelectric generation system for waste heat recovery. Renewable Energy.36(2011) 3530-3536.

[11] Xiaolong Gou, Suwen Yang, Heng Xiao, Qiang Ou. A dynamic model for thermoelectric generator applied in waste heat recovery.Energy. 52(2013) 201-209.

[12] R.O. Suzuki. Mathematic simulation on power generation by roll cake type of thermoelectric double cylinders. J. Power Sources, 133 (2004) 277-285.

[13] C. Baker, P. Vuppuluri, L. Shi, et al. Model of heat exchangers for waste heat recovery from diesel engine exhaust for thermoelectric power generation. J. Electron. Mater., 41 (2012) $1290-1297$.

[14] S. Krishnan, N. Karri, P. Gogna, et al. Progress towards an optimization methodology for combustion-driven portable thermoelectric power generation systems. J. Electron. Mater., 41 (2012) 1622-1631.

[15] B. Orr, B. Singh, L. Tan, et al. Electricity generation from an exhaust heat recovery system utilizing thermoelectric cells and heat pipes.Appl. Therm. Eng. 73(2014)588-597.

[16] Qungui Du, Jiehui Zou, Shuijin Chen, Junling Gao. Calculation Model of Semiconductor Thermoelectric Generator Unit Based on Variable Material Properties. Journal of South China University of Technology(Natural Science Edition). 41(2013) 47-53.(In Chinese) 